\title{
Neuroprotective effect of diosgenin in a mouse model of diabetic peripheral neuropathy involves the Nrf2/HO-1 pathway
}

Jinhong Leng ${ }^{1+}$, Xiaohua $\mathrm{Li}^{2+}$, He Tian ${ }^{3^{*}}$, Chang Liu ${ }^{4 *}$, Yining Guo ${ }^{2}$, Su Zhang ${ }^{2}$, Yang Chu ${ }^{2}$, Jian $\mathrm{Li}^{2}$, Ying Wang ${ }^{2}$ and Ling Zhang ${ }^{2}$

\begin{abstract}
Background: Diabetic peripheral neuropathy (DPN) is one of the most common chronic complications of diabetes. Diosgenin is a natural steroidal saponin with a variety of beneficial effects, including antidiabetic effects, and is a raw material for the synthesis of carrier hormones. In our study, we aimed to assess the antioxidant effects of diosgenin in diabetic mice.

Methods: Male C57 mice were fed a high-fat diet for 8 weeks and intraperitoneally injected with streptozotocin (STZ) at a dose of $100 \mathrm{mg} / \mathrm{kg}$ for 2 consecutive days. Eligible mice were divided into the normal control group (CON), diabetic group (DM), low-dose diosgenin (50 mg/kg) group (DIO50) and high-dose diosgenin (100 mg/kg) group (DIO100). Treatment was started 6 weeks after the induction of diabetes by STZ and continued for 8 weeks. Blood sugar and body weight were monitored dynamically. The behavioural effects of diosgenin were detected by a hot tail immersion test and paw tactile responses. HE staining was used to evaluate edema and degeneration of the sciatic nerve. The levels of SOD, MDA and GPx were tested according to the instructions of the respective kits. The levels of Nrf2, HO-1 and NQO1 were detected by immunofluorescence and Western blotting. Statistical analysis was performed using SPSS, and $P<0.05$ was considered statistically significant.
\end{abstract}

Results: Diosgenin decreased the blood glucose levels and increased the body weight of diabetic mice. There was a significant increase in the tail withdrawal latency of diabetic animals, and mechanical hyperalgesia was significantly alleviated after diosgenin treatment. Histopathological micrographs of HE-stained sciatic nerves showed improvement after diosgenin treatment. Diosgenin attenuated the level of MDA but increased the activities of SOD and GPx. Diosgenin increased the expression of Nrf2, HO-1 and NQO1.

Conclusions: Our results demonstrate that diosgenin can ameliorate behavioural and morphological changes in DPN by reducing oxidative stress. The Nrf2/HO-1 signalling pathway was involved in its neuroprotective effects.

Keywords: Diosgenin, Diabetic peripheral neuropathy, Nrf2, HO-1, Oxidative stress

\footnotetext{
* Correspondence: jytianhe@foxmail.com; liuchang1971mei@163.com

${ }^{\dagger}$ Jinhong Leng and Xiaohua Li contributed equally to this work.

${ }^{3}$ Department of Histology and Embryology, School of Basic Medicine, Jinzhou Medical University, Jinzhou 121000, Liaoning, China

${ }^{4}$ Department of Endocrinology, The First Affiliated Hospital of Jinzhou Medical University, Jinzhou 121000, Liaoning, China

Full list of author information is available at the end of the article
}

(c) The Author(s). 2020 Open Access This article is licensed under a Creative Commons Attribution 4.0 International License, which permits use, sharing, adaptation, distribution and reproduction in any medium or format, as long as you give appropriate credit to the original author(s) and the source, provide a link to the Creative Commons licence, and indicate if changes were made. The images or other third party material in this article are included in the article's Creative Commons licence, unless indicated otherwise in a credit line to the material. If material is not included in the article's Creative Commons licence and your intended use is not permitted by statutory regulation or exceeds the permitted use, you will need to obtain permission directly from the copyright holder. To view a copy of this licence, visit http://creativecommons.org/licenses/by/4.0/. The Creative Commons Public Domain Dedication waiver (http://creativecommons.org/publicdomain/zero/1.0/) applies to the data made available in this article, unless otherwise stated in a credit line to the data. 


\section{Background}

Diabetes is a common and complex endocrine disease that can cause serious complications in multiple tissues, and it has become a serious public health problem worldwide [1, 2]. Diabetic neuropathy is an important factor leading to disability in diabetic patients [3]. It is estimated that in 2015, there were 415 million adults worldwide suffering from diabetes; additionally there are many undiagnosed adults who suffer from impaired glucose tolerance, which is a major risk factor for diabetes [4]. There is a common complication in people with diabetes that is characterized by greater sensitivity to noxious stimuli than that of normal people (hyperalgesia) [5]. Clinically, the symptoms of diabetic peripheral neuropathy seriously affect the quality of life and mental health of patients, so treating these symptoms of DPN in the clinic is a new challenge [6]. Currently, there are very few available therapies for diabetic neuropathy because therapeutic opportunities are limited by many factors, such as serious adverse reactions and ineffectiveness. Therefore, we still need to find a suitable treatment for these complications of neuropathy [7].

Chronic hyperglycaemia develops, oxidative stress is activated, and a series of complex reactions lead to nerve tissue damage, which in turn causes neuropathic pain [8]. There are many opinions on the pathogenesis of diabetic neuropathy. Early reports highlighted the importance of four hyperglycaemic-mediated cellular pathways, including the protein kinase $\mathrm{C}$ (PKC), advanced glycation end product (AGE), polyol and hexosamine pathways [9]. Later, it was discovered that these pathways are linked by oxidative-nitrosative stress and that oxidativenitrosative stress is related in some way to all known aetiologies of diabetic neuropathy [10]. Oxidative stress is one of the main potential mechanisms of painful diabetic peripheral neuropathies. Oxidative stress can lead to neurological damage in a variety of neuropathies, including diabetic neuropathy, Charcot-Marie neuropathy, and acrylamide-induced neuropathy [11-14]. Therefore, we assessed changes in oxidative stress our study of DPN.

Neurons have their own defence system to resist oxidative stress, which includes various enzymatic antioxidant and nonenzymatic antioxidants (superoxide dismutase (SOD), catalase, glutathione S-transferase (GST), glutathione peroxidase (Gpx), glutathione (GSH), various vitamins, etc.) that detoxify reactive oxygen species (ROS) and reduce nerve damage, but this defence system is very weak. In the case of chronic hyperglycaemia, the redox balance in the body is disrupted, resulting in damage to proteins, DNA and cell membranes, which ultimately leads to the impairment of neuronal function $[11,15]$.
Nrf2 is an important transcription factor that regulates cellular oxidative stress. It is beneficial for ameliorating oxidative stress, promoting cell survival and maintaining redox homeostasis in cells. The Nrf2-ARE signalling pathway initiates the regulation of its detoxification enzymes, such as nucleotide adenosine diphosphate hydrogenase (NADPH), haem oxygenase-1 (HO-1) and quinone oxidoreductase-1 (NQO1), which resist oxidative stress and protect cells [16].

At present, it is necessary to design an effective new compound for relieving pain in diabetic peripheral neuropathy. Chinese herbal plants and their active ingredients are used to manage diabetes mellitus and its complications [17, 18]. Diosgenin is the main component of the Chinese herbal medicine Dioscorea nipponica Makino, which is a steroidal saponin in the form of glycoside $[19,20]$. It has been found to have many beneficial effects, including hypoglycaemic, [21, 22], cardiovascular protective [21, 23] and hypolipidaemic effects [24]. Diosgenin can also attenuate oxidative damage induced by $\mathrm{D}$-galactose in ageing mice [25]. It also enhances the antioxidant defence system, relieves oxidative stress, and reduces inflammation or apoptosis [23]. Therefore, we hypothesize that diosgenin may prevent or ameliorate diabetic peripheral neuropathy.

Streptozotocin (STZ)-induced diabetic rats develop symptoms of hyperalgesia after being exposed to harmful external stimuli, so they are often used as a model of diabetic peripheral neuropathy and can also be used to test the effect of analgesic drugs [26-28]. In this study, we used male C57 mice with STZ-induced diabetes to evaluate the protective effects of diosgenin on neuropathy in diabetic mice. Behavioural testing and measurements of biochemical markers associated with oxidative stress in sciatic nerves were performed.

\section{Methods \\ Animals and treatment}

Eight-week-old male C57 mice were purchased from the Laboratory Animal Center of Jinzhou Medical University. The mice were housed under a 12-h light-dark cycle, food and water were freely available. All procedures were conducted in accordance with the ethical guidelines set up by the International Association for the Study of Pain (IASP) on the use of laboratory animals in experimental research. The animal study was approved by the Animal Care and Use Committee of Jinzhou Medical University.

After being fed a high-fat diet (Casein, Lactic, 30 Mesh:200 g; Cystine, L: 3 g; Sucrose, Fine Granulated: 354 g; Starch, Corn: 315 g; Lodex 10: 35 g; Solka Floc, FCC200: 50 g; Soybean Oil, USP: 25 g; Lard: 20 g; S10026B: 50 g; Choline Bitartrate: 2 g; V10001C: 1 g; Dye, Yellow FD\&C \#5, Alum. Lake 35-42\%: 0.05 g; 
Total: $1055.05 \mathrm{~g}$ ) for 8 weeks, the mice were randomly assigned to groups and fasted for $12 \mathrm{~h}$. Then, diabetes was induced by intraperitoneal (i.p.) injection of $100 \mathrm{mg} /$ kg STZ (Sigma, USA) for 2 consecutive days. Immediately prior to injection, STZ was dissolved in sodium citrate buffer ( $\mathrm{pH} 4.5)$. The control mice were intraperitoneally injected with the same dose of sodium citrate buffer. Blood glucose levels in samples from the tail vein were monitored weekly. Weight was evaluated every week. Animals that exhibited blood glucose levels $>16.7 \mathrm{mmol} / \mathrm{L}$ within 1 week after the completion of STZ injection were considered diabetic and were included in the study; these animals showed progression to neuropathy-like symptoms and were tested behaviourally after 6 weeks $[29,30]$.

Eligible mice were divided into the normal control group (CON), diabetic group (DM), low-dose diosgenin $(50 \mathrm{mg} / \mathrm{kg})$ group (DIO50) and high-dose diosgenin $(100 \mathrm{mg} / \mathrm{kg})$ group (DIO100) [31]. There were six mice per group, and additional mice were added in the case of death. Diosgenin (Molecular formula: C27H42O3, Molecular weight:414.627. Solarbio Science \& Technology Co., Ltd.,) was dissolved in 0.5\% CMC-Na solution. The drug was administered intragastrically. The control group was given the same dose of vehicle. Treatment was started 6 weeks after the induction of diabetes by STZ diabetes and continued for 8 weeks. The body weight and blood glucose levels of the mice were monitored continuously. Behaviour and related parameters were tested $24 \mathrm{~h}$ after the last intragastric administration.

\section{Behavioural tests}

Before starting the behavioural tests, the mice were habituated to the experimental site for $1 \mathrm{~h}$ to prevent the environment from affecting behavioural responses. Behavioural testing was performed $24 \mathrm{~h}$ after the last intragastric administration of the drug. To test the behavioural effects of diosgenin in diabetic mice, we used the hot tail immersion test and the Von Frey test.

\section{Hot tail immersion test}

To detect thermal hyperalgesia in mice, warm water with a temperature of $50 \pm 0.5^{\circ} \mathrm{C}$ was prepared. The mice were gently restrained with a towel, and the tail was exposed. One third of the tail was quickly immersed in the water, and a stopwatch was used to record the duration of tail immersion and the time at which the tail flick reflex began. The cut-off time was set at $30 \mathrm{~s}$ to prevent tissue damage. The experiment was repeated 4 times per mouse, and there was an experimental interval of $5 \mathrm{~min}$. The final results were averaged [32].

\section{Paw tactile response test}

To detect tactile allodynia in the mice, the Von Frey test was performed. The mice were placed in a cage with a stainless-steel mesh bottom for at least $15 \mathrm{~min}$. A series of Von Frey filaments (range: 0.008-300 g) were then gradually applied vertically to the left hind paw of each mouse so that the filament flexed. Lifting, shaking, or licking of the paws were considered positive reactions. Each mouse was tested 3 times with an interval of at least $10 \mathrm{~min}$. The final results were averaged [32].

\section{Tissue sample collection}

At the end of the experiments, the mice were sacrificed by cervical dislocation under anaesthesia with $3 \%$ sodium pentobarbital by intraperitoneal injection. Three mice from each group were fixed with $4 \%$ paraformaldehyde via arterial perfusion. The sciatic nerves were isolated quickly and stored in 4\% paraformaldehyde solution for tissue slicing. The sciatic nerves of the other three mice in each group were isolated quickly and stored at $-80^{\circ} \mathrm{C}$.

\section{Haematoxylin-eosin staining}

The sciatic nerves stored in $4 \%$ paraformaldehyde were dehydrated and embedded in paraffin and then cut at a thickness of $5 \mu \mathrm{m}$ to prepare slices. The sections were stained with haematoxylin and eosin (H\&E), sealed with gum, air-dried and observed under a light microscope (magnification: $40 \times$ ) to evaluate edema and degeneration of the sciatic nerve.

\section{Evaluation of SOD, MDA and GPX}

The levels of SOD, MDA and GPx in the sciatic nerves of the mice were tested according to the instructions of the respective kits (Jiancheng Bioengineering Institute, China).

\section{Western blot}

The sciatic nerves stored at $-80^{\circ} \mathrm{C}$ were placed in prepared RIPA lysis buffer, shredded, sonicated, fully lysed, and centrifuged, and the supernatant was collected for quantitative protein analysis. Then, the samples were diluted to the same protein concentration. Equal amounts of proteins were separated by SDS-PAGE and transferred to a PVDF membrane. After blocking with $3 \%$ BSA, the membrane was incubated with primary antibodies against Nrf2, HO-1 and NQO1 (Thermo Scientific, USA) overnight at $4{ }^{\circ} \mathrm{C}$. Then, the cells were washed three times with TBST for 5 min each time. The membrane was incubated for $2 \mathrm{~h}$ with the corresponding secondary antibody (Thermo Scientific). The bound antibodies were visualized using a Fusion Chemiluminescence Imager. The relative band densities were quantified by densitometry using ImageJ software. 


\section{Immunofluorescence analysis}

The sciatic nerve sections were washed three times with PBS for $5 \mathrm{~min}$ at room temperature. They were then permeabilized with $3 \%$ Triton-X 100 for $5 \mathrm{~min}$ and washed three times with PBS. They were then blocked with goat serum for $2 \mathrm{~h}$ and incubated overnight at $4{ }^{\circ} \mathrm{C}$ with an Nrf2 primary antibody. The staining methods for HO-1 and NQO1 were the same. The next day, the sections were washed three times with PBS for $5 \mathrm{~min}$ each and incubated with a secondary antibody directed against the primary antibody for $2 \mathrm{~h}$, after which they were protected from light. The sections were washed three times with PBS, incubated with DAPI, and covered with coverslips. The slides were observed using a fluorescence microscope.

\section{Statistical analysis}

All the obtained data are expressed as the mean \pm SEM, and statistical analysis was performed using SPSS version 22.0. The parameters were analysed by using one-way ANOVA followed by least significant difference (LSD) post hoc test. $P<0.05$ was considered statistically significant.

\section{Results}

\section{Diosgenin decreased the blood glucose levels and} increased the body weight of diabetic mice

During the study period, vehicle-treated diabetic mice showed a significant decrease in body weight at week 8 , and compared with the vehicle-treated diabetic mice, the treated mice showed improvements in body weight after the intragastric administration of the two doses of diosgenin for 8 weeks (Fig. 1a). In addition, the diabetic group exhibited a significant increase in blood glucose levels at week 8 compared with week 0 , and compared with vehicle-treated diabetic mice, diabetic mice treated with diosgenin presented significantly reduced blood glucose levels at week 8 (Fig. 1b).

\section{Diosgenin reduced hyperalgesia and allodynia in diabetic mice}

In the hot immersion test, the tail-flick latency of the diabetic animals was significantly lower than that of the normal control animals $(P<0.01)$. There was a significant increase in the tail withdrawal latency of diabetic animals treated with the two doses of diosgenin $(P<$ 0.01 ) (Fig. 2a). Mechanical hyperalgesia was more pronounced in diabetic animals than in normal control animals. The Von Frey test showed that mechanical hyperalgesia was apparent in diabetic animals $(P<0.01)$, and this hyperalgesia was significantly alleviated after diosgenin treatment $(P<0.01)$ (Fig. $2 \mathrm{~b})$.

\section{Diosgenin administration improved histopathological changes in the sciatic nerves of diabetic mice}

Histopathological micrographs showed significant axonal degeneration, myelinolysis, and endometrial edema in the sciatic nerves of DM animals compared to those of the normal control group. There were improvements in axonal degeneration, myelinolysis and endometrial edema in the mice treated with diosgenin (Fig. 3).

\section{Diosgenin administration reduced oxidative stress in the sciatic nerve of diabetic mice}

The level of MDA (a marker of oxidative stress) in the sciatic nerves of diabetic mice was elevated compared with that in the sciatic nerves of normal mice $(P<0.01)$. Diosgenin attenuated the level of MDA, and this effect was dose-dependent (Fig. 4a). The antioxidant enzymes SOD and GPx were inhibited in the sciatic nerves of diabetic mice. The two different doses of diosgenin both increased the activity of antioxidant enzymes (Fig. 4b-c). The results indicated that the administration of

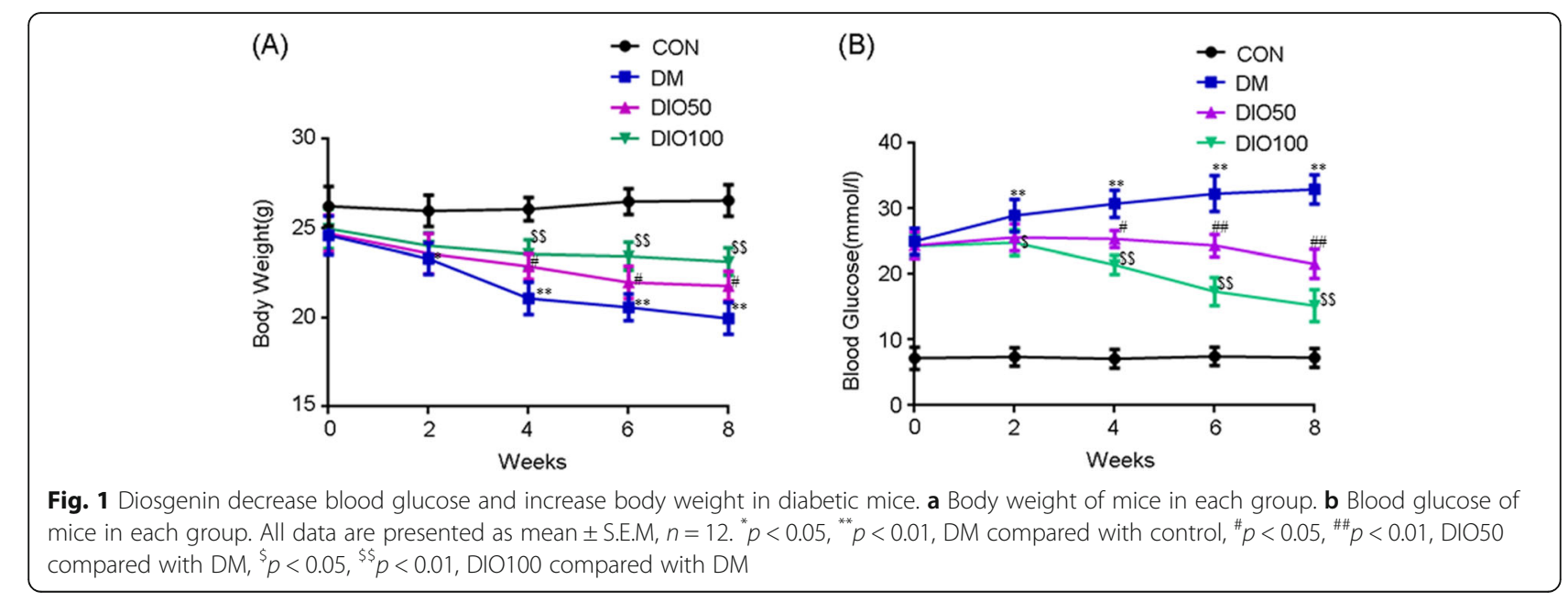



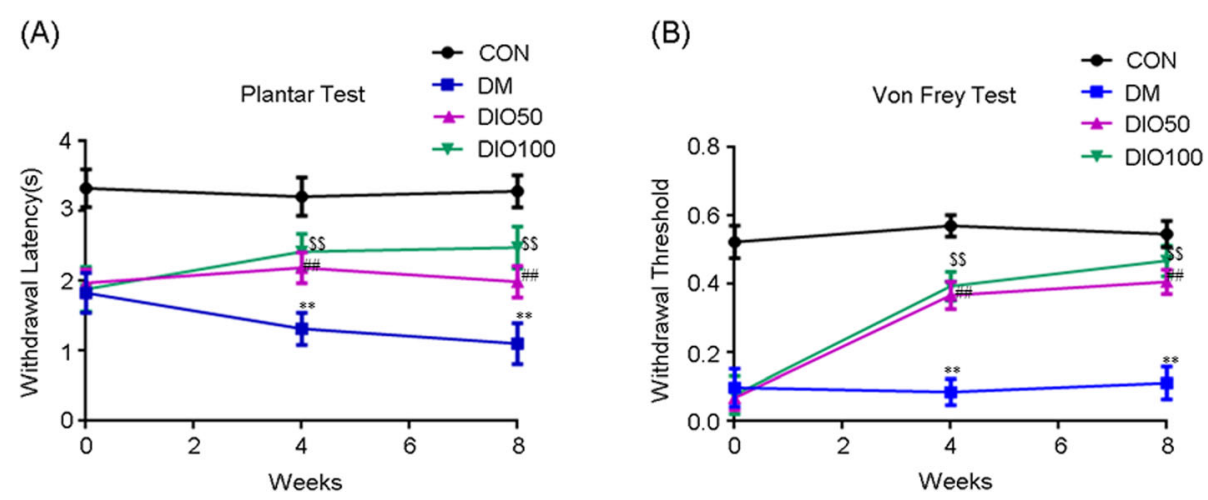

Fig. 2 The effect of diosgenin treatment on behavioral performance in diabetic mice. a Thermal nociceptive threshold of hot tail immersion test. b Mechanical nociceptive threshold of Von Frey test. All data are presented as mean \pm S.E.M, $n=12^{* *} p<0.01$, DM compared with control, ${ }^{\# \#} p<$ 0.01, DIO50 compared with DM, ${ }^{\$} p<0.01$, DIO100 compared with DM

diosgenin can restore the beneficial effects of the antioxidant defence system in diabetic mice.

\section{Diosgenin protected the sciatic nerves of diabetic mice through the Nrf2/HO-1 pathway}

To explore the mechanism by which diosgenin resists oxidative stress, the levels of proteins associated with the Nrf2/HO-1 pathway were detected. The results of immunofluorescence staining showed that the expression of Nrf2, HO-1 and NQO1 was decreased significantly in the sciatic nerves of diabetic mice compared to those of normal control mice. However, the fluorescence intensities of Nrf2, HO-1 and NQO1 were increased after the administration of diosgenin (Fig. 5). The Western blot results were consistent with the immunofluorescence staining results. There was a significant decrease in Nrf2 expression in diabetic sciatic nerve compared to normal sciatic nerves $(P<0.05)$. Moreover, decreases in the levels of the downstream cell protective enzymes HO-1 and NQO1 were detected $(\mathrm{P}<0.05)$. The administration of $50 \mathrm{mg} / \mathrm{kg}$ and $100 \mathrm{mg} / \mathrm{kg}$ diosgenin increased the level of $\mathrm{Nrf2}$ in diabetic mice and increased the levels of $\mathrm{HO}-1$ and NQO1 $(P<0.01)$ (Fig. 6).

\section{Discussion}

DPN is one of the most common complications in diabetic patients and is characterized by disruption of nerve conduction in the peripheral nervous system [33]. The common pathogeneses of DPN are inflammatory damage to myelinated neurons and oxidative stress in endothelial cells [8]. It has been observed in clinical and experimental DPN that the innervation of peripheral nerve tissue is decreased. Because of hypoxia in the endometrium, neuroischaemia, the loss of neurotrophic support, and neurological dysfunction are observed [34]. Studies have shown that a variety of mechanisms, such as adrenergic mechanisms, Na1 currents, opioid neurotransmission and oxidative stress, are involved in the neuropathic pain response in DPN [35-37]. In recent years, many researchers have focused on oxidative stress in peripheral neuropathy. It is an important factor in

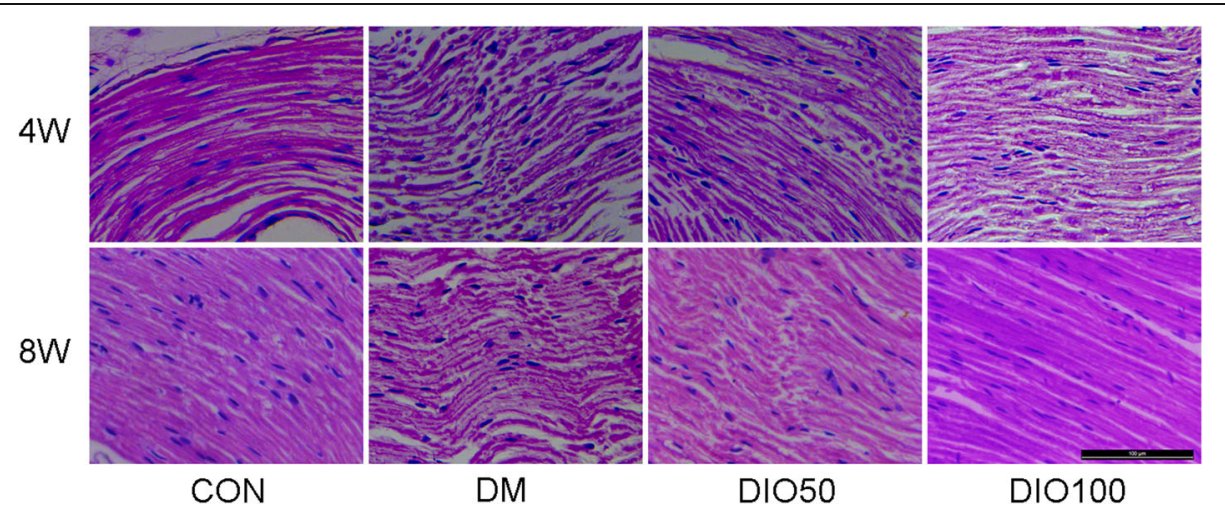

Fig. 3 Images of H\&E staining of sciatic nerves of mice in each group. In the mice treated with diosgenin $50 \mathrm{mg} / \mathrm{kg}$ or $100 \mathrm{mg} / \mathrm{kg}$ for $4 \mathrm{~W}$ or $8 \mathrm{~W}$, there was improvement with sciatic nerve histopathological changes 


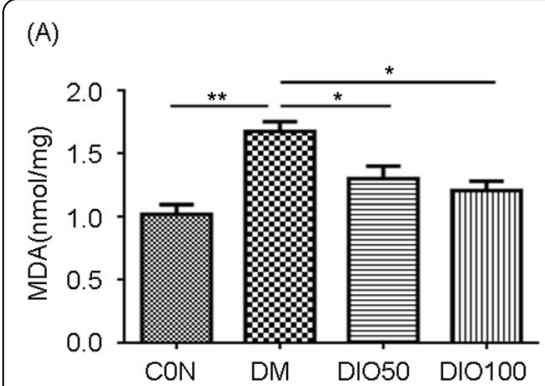

(B)

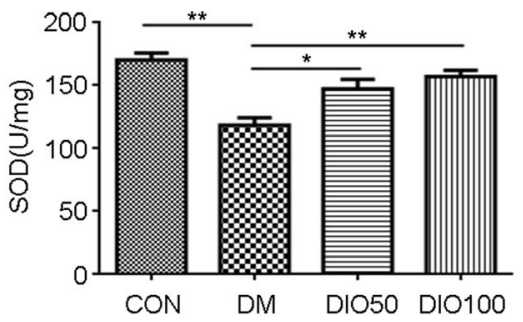

(C)

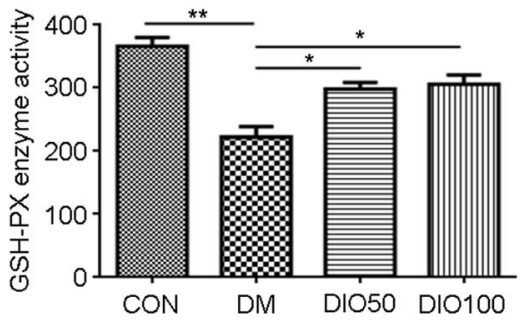

Fig. 4 Diosgenin administration reduced oxidative stress in sciatic nerve of diabetic mice. a The level of MDA in sciatic nerve. $\mathbf{b}$ The level of SOD in sciatic nerve. c The level of GPX in sciatic nerve. All data are presented as mean \pm S. E.M. $(n=3),{ }^{*} p<0.05,{ }^{* *} p<0.01$

peripheral neuropathy caused by chemotherapy and diabetic neuropathy [11, 38]. We conclude from this study that the neuroprotective effects of diosgenin are mainly exerted through a reduction in the oxidative stress response and the enhancement of the oxidative defence system. The results confirmed previous findings regarding the therapeutic effects of diosgenin $[39,40]$.

Histological analysis showed that the biochemical changes in STZ-induced diabetic mice included degeneration of sciatic nerve fibres and endometrial edema. The sciatic nerves of mice treated with diosgenin showed almost normal axons and intact myelin. The beneficial effects of diosgenin observed by histology may be related to its antioxidant capacity. In summary, diosgenin treatment improves tissue changes in the sciatic nerve, possibly due to its antioxidant capacity.

It has been determined that oxidative stress is a major factor in diabetic neuropathy and leads to an abnormal pain response in DPN [8]. The increase of MDA, TBARS, and isoprostanes have been observed in diabetes experimental model $[41,42]$. Damage to the antioxidant defence system and hyperoxia cause peripheral nerves to be susceptible to oxidative damage, and neuropathy in diabetic animals can be alleviated by minimizing

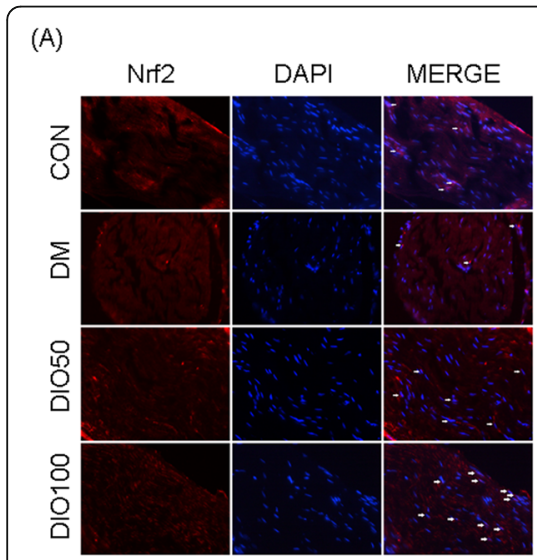

(D)

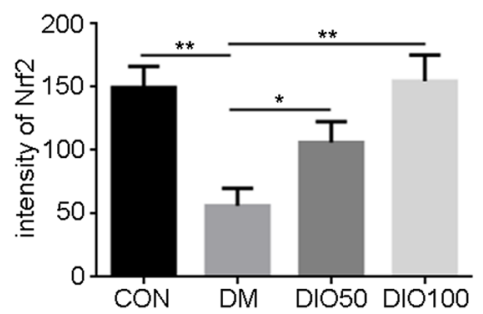

(B)

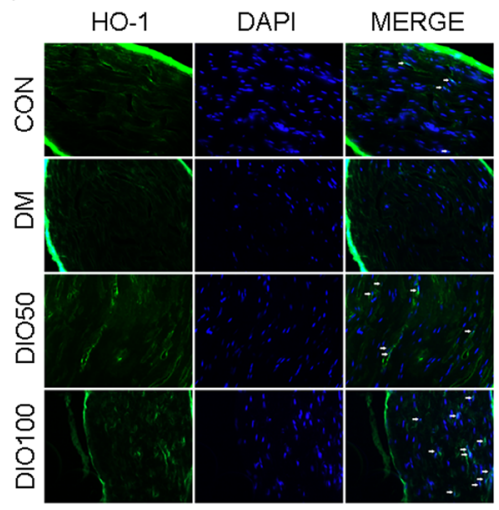

(E)

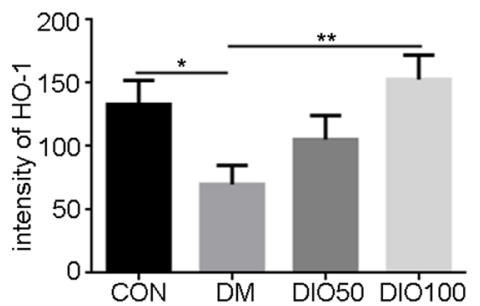

(C)

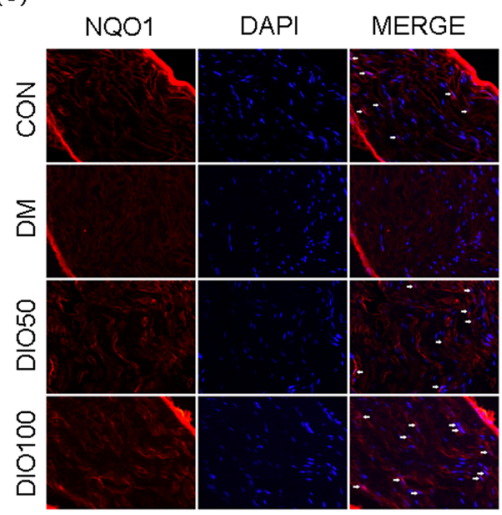

$(\mathrm{F})$

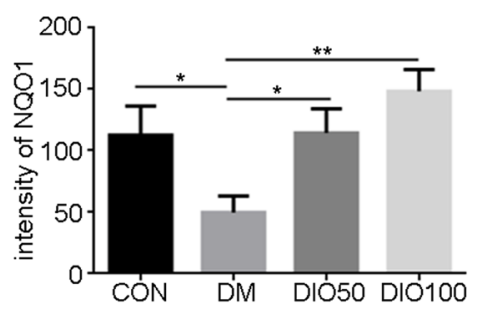

Fig. 5 Expression levels of Nrf2, $\mathrm{HO}-1$ and $\mathrm{NQO} 1$ in sciatic nerves. Immunofluorescence results of $\mathrm{Nrf2}$ (a), HO-1 (b) and NQO1 (c) in sciatic nerves from different groups. $\mathbf{d}$ The fluorescence intensity of Nrf2. e The fluorescence intensity of HO-1. $\mathbf{f}$ The fluorescence intensity of NQO1. All data are presented as mean \pm S.E.M. $(n=3),{ }^{*} p<0.05, \quad p<0.01$ 

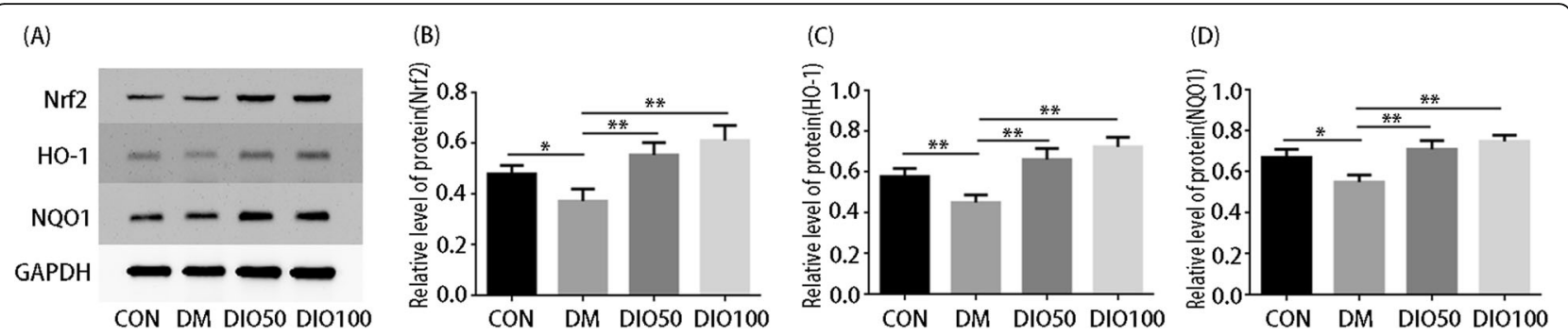

Fig. 6 Effect of diosgenin on the Nrf2/ HO-1 signaling pathway. a Immunoblot analyses of the protein levels of Nrf2, HO-1 and NQO1 in sciatic nerves treated with diosgenin $50 \mathrm{mg} / \mathrm{kg}$ or $100 \mathrm{mg} / \mathrm{kg}$. b-d Administration of diosgenin increased the protein levels of Nrf2 in diabetic mice, and also increased the levels of HO-1 and NQO1. All data are presented as mean \pm S.E.M. $(n=3) .{ }^{*} p<0.05,{ }^{* *} p<0.01$

oxidative damage in the peripheral nerves [43]. ROS can cause damage to peripheral nerves by increasing oxidative stress or attenuating the antioxidant defence systems [11]. They can cause DNA damage and cellular oxidation reactions such as protein oxidation and cell membrane lipid peroxidation [44, 45]. However, the cells themselves have enzymatic and nonenzymatic antioxidant defence systems that detoxify ROS, such as SOD and GPx. However, chronic stress in DPN destroys the antioxidant capacity of cells, leading to the development of abnormal oxidative stress and molecular changes associated with DPN [46]. Previous studies have identified glucose-induced superoxide production as an important part of the pathophysiology of diabetic microvascular complications [10]. High doses of glucose cause NADH flux to increase the intensity of free radical production, which further leads to a series of reactions, such as protein nitration at tyrosine residues and DNA damage [47]. In the present study, the production of MDA by the sciatic nerves was increased in diabetic mice compared with normal control, indicating an increase in oxidative stress. But, diosgenin could ameliorated MDA. Our results also showed that diosgenin significantly increased the levels of SOD and GPx in sciatic nerves. In our study, several changes in the levels of oxidative stress confirmed that diosgenin inhibited oxidative stress in diabetic mice. The reduction in oxidative stress in the sciatic nerve may be attributed to the antinociceptive effect of diosgenin.

Nrf2 is a critical transcription factor of the antioxidant defence system that induces the expression of phase II detoxification enzymes (HO-1, NQO1 and epoxide hydrolase, etc.) [48]. HO-1 has also been found to have potential neurovascular protective properties in diabetic neuropathy [49]. In our study, in the presence of high glucose, DPN mice showed a decrease in the level of $\mathrm{Nrf} 2$, which in turn led to decreases in the levels of $\mathrm{HO}-1$ and NQO1. After the administration of diosgenin, the expression of $\mathrm{Nrf} 2$ increased in mice, and the trend of HO-1 and NQO1 expression was the same. This shows the beneficial effect of diosgenin on the antioxidant defence system.

\section{Conclusion}

This study focused on improvements in neuropathy elicited by diosgenin in STZ-induced diabetic mice. Our results demonstrate that diosgenin can ameliorate the behavioural and morphological changes observed in DPN by reducing oxidative stress. The Nrf2/HO-1 signalling pathway is involved in the neuroprotective effects of diosgenin.

\section{Acknowledgements \\ Not applicable.}

\section{Authors' contributions}

$\mathrm{J}-\mathrm{HL}$ and $\mathrm{CL}$ designed the study. $\mathrm{X}-\mathrm{HL}$ interpreted the experimental data and wrote the paper. HT designed the research and revised the manuscript. Y-NG and $\mathrm{SZ}$ carried out the animal experiments. $\mathrm{YC}$ and $\mathrm{J}$ performed the morphological experiments. YW and LZ contributed to the molecular biology experiments. All authors have read and approved the manuscript.

\section{Funding}

This study was funded by the Natural Science Foundation of Liaoning Province (20180530030 and 20180550649) and the Science and Technology Planning Project of Shenyang (18-013-0-09). This work was also supported by the Liaoning BaiQianWan Talents Program. The funding was used to defray the cost of the animals and reagents.

\section{Availability of data and materials}

The raw data for this study are available upon reasonable request to the corresponding author.

\section{Ethics approval and consent to participate}

Experiments were conducted in accordance with the ethical guidelines set up by the International Association for the Study of Pain (IASP) on the use of laboratory animals in experimental research. The animal study was approved by the Animal Care and Use Committee of Jinzhou Medical University.

\section{Consent for publication}

Not applicable.

\section{Competing interests}

The authors declare that they have no competing interests.

\section{Author details}

'Department of Endocrinology, Affiliated Hospital of Liaoning University of Traditional Chinese Medicine, Shenyang 110032, Liaoning, China.

${ }^{2}$ Department of Traditional Chinese Medicine Clinical Endocrinology, Liaoning University of Traditional Chinese Medicine Graduate School, Shenyang 110847, Liaoning, China. ${ }^{3}$ Department of Histology and 
Embryology, School of Basic Medicine, Jinzhou Medical University, Jinzhou 121000, Liaoning, China. ${ }^{4}$ Department of Endocrinology, The First Affiliated Hospital of Jinzhou Medical University, Jinzhou 121000, Liaoning, China.

\section{Received: 12 November 2019 Accepted: 16 April 2020 \\ Published online: 26 April 2020}

\section{References}

1. Gupta G, de Jesus Andreoli Pinto T, Chellappan DK, Mishra A, Malipeddi H, Dua K. A clinical update on metformin and lung cancer in diabetic patients. Panminerva Med. 2018:60(2):70-5.

2. Kiasalari Z, Rahmani T, Mahmoudi N, Baluchnejadmojarad T, Roghani M. Diosgenin ameliorates development of neuropathic pain in diabetic rats: involvement of oxidative stress and inflammation. Biomed Pharmacother. 2017:86:654-61.

3. Didangelos T, Doupis J, Veves A. Painful diabetic neuropathy: clinical aspects. Handbook Clin Neurol. 2014;126:53-61.

4. Ogurtsova K, da Rocha Fernandes JD, Huang Y, Linnenkamp U, Guariguata L, Cho NH, Cavan D, Shaw JE, Makaroff LE. IDF diabetes atlas: global estimates for the prevalence of diabetes for 2015 and 2040. Diabetes Res Clin Pract. 2017;128:40-50.

5. Alshahrani S, Fernandez-Conti F, Araujo A, DiFulvio M. Rapid determination of the thermal nociceptive threshold in diabetic rats. J Vis Exp. 2012;63:e3785.

6. Degu H, Wondimagegnehu A, Yifru YM, Belachew A. Is health related quality of life influenced by diabetic neuropathic pain among type ॥ diabetes mellitus patients in Ethiopia? PLoS One. 2019;14(2):e0211449.

7. Bachewal P, Gundu C, Yerra VG, Kalvala AK, Areti A, Kumar A. Morin exerts neuroprotection via attenuation of ROS induced oxidative damage and neuroinflammation in experimental diabetic neuropathy. Biofactors. 2018; 44(2):109-22.

8. Sandireddy R, Yerra VG, Areti A, Komirishetty P, Kumar A. Neuroinflammation and oxidative stress in diabetic neuropathy: futuristic strategies based on these targets. Int J Endocrinol. 2014:2014:674987.

9. Tomlinson DR, Gardiner NJ. Glucose neurotoxicity. Nat Rev Neurosci. 2008; 9(1):36-45.

10. Brownlee M. The pathobiology of diabetic complications: a unifying mechanism. Diabetes. 2005:54(6):1615-25.

11. Negi G, Kumar A, Joshi RP, Sharma SS. Oxidative stress and Nrf2 in the pathophysiology of diabetic neuropathy: old perspective with a new angle. Biochem Biophys Res Commun. 2011;408(1):1-5

12. Negi G, Kumar A, Sharma SS. Melatonin modulates neuroinflammation and oxidative stress in experimental diabetic neuropathy: effects on NF-KB and Nrf2 cascades. J Pineal Res. 2011;50(2):124-31.

13. Prasad SN, Muralidhara X. Neuroprotective effect of geraniol and curcumin in an acrylamide model of neurotoxicity in Drosophila melanogaster: relevance to neuropathy. J Insect Physiol. 2014;60:7-16.

14. Saifi GM, Szigeti K, Snipes GJ, Garcia CA, Lupski JR. Molecular mechanisms, diagnosis, and rational approaches to management of and therapy for Charcot-Marie-tooth disease and related peripheral neuropathies. J Invest Med. 2003;51(5):261-83.

15. Feldman EL. Oxidative stress and diabetic neuropathy: a new understanding of an old problem. J Clin Investig. 2003;111(4):431-3.

16. Wang $W$, Wu $Y$, Z Zhang $G$, Fang $H$, Wang $H$, Zang $H$, Xie $T$, Wang $W$. Activation of Nrf2-ARE signal pathway protects the brain from damage induced by epileptic seizure. Brain Res. 2014;1544:54-61.

17. You LZ, Lin YX, Fang ZH, Shen GM, Zhao JD, Wang TT. Research advances on astragaloside-IV in treatment of diabetes mellitus and its complications pharmacological effects. Zhongguo Zhong Yao Za Zhi. 2017;42(24):4700-6.

18. Xue B, Wang L, Zhang Z, Wang R, Xia XX, Han PP, Cao LJ, Liu YH, Sun LQ. Puerarin may protect against Schwann cell damage induced by glucose fluctuation. J Nat Med. 2017:71(3):472-81.

19. Chen Y, Tang YM, Yu SL, Han YW, Kou JP, Liu BL, Yu BY. Advances in the pharmacological activities and mechanisms of diosgenin. Chin J Nat Med. 2015;13(8):578-87.

20. Liu W, Zhao Z, Wang Y, Li W, Su Q, Jia Q, Zhang J, Zhang X, Shen J, Yin J. Dioscin inhibits stem-cell-like properties and tumor growth of osteosarcoma through Akt/GSK3/ $\beta$-catenin signaling pathway. Cell Death Dis. 2018;9(3):343.

21. Roghani-Dehkordi F, Roghani M, Baluchnejadmojarad T. Diosgenin mitigates Streptozotocin diabetes-induced vascular dysfunction of the rat aorta: the involved mechanisms. J Cardiovasc Pharmacol. 2015;66(6):584-92.
22. Saravanan G, Ponmurugan P, Deepa MA, Senthilkumar B. Modulatory effects of diosgenin on attenuating the key enzymes activities of carbohydrate metabolism and glycogen content in streptozotocin-induced diabetic rats. Can J Diabetes. 2014:38(6):409-14.

23. Ahmed LA, Obaid AA, Zaki HF, Agha AM. Role of oxidative stress, inflammation, nitric oxide and transforming growth factor-beta in the protective effect of diosgenin in monocrotaline-induced pulmonary hypertension in rats. Eur J Pharmacol. 2014;740:379-87.

24. LV YC, Yang J, Yao F, Xie W, Tang YY, Ouyang XP, He PP, Tan YL, Li L, Zhang $M$, et al. Diosgenin inhibits atherosclerosis via suppressing the MiR-19binduced downregulation of ATP-binding cassette transporter A1. Atherosclerosis. 2015;240(1):80-9.

25. Chiu CS, Chiu YJ, Wu LY, Lu TC, Huang TH, Hsieh MT, Lu CY, Peng WH. Diosgenin ameliorates cognition deficit and attenuates oxidative damage in senescent mice induced by D-galactose. Am J Chin Med. 2011;39(3):551-63.

26. Baluchnejadmojarad T, Roghani M, Roghani-Dehkordi F. Antinociceptive effect of Teucrium polium leaf extract in the diabetic rat formalin test. Ethnopharmacol. 2005;97(2):207-10.

27. Mirshekar M, Roghani M, Khalili M, Baluchnejadmojarad T, Arab MS. Chronic oral pelargonidin alleviates streptozotocin-induced diabetic neuropathic hyperalgesia in rat: involvement of oxidative stress. Iran Biomed J. 2010; 14(1-2):33-9.

28. Baluchnejadmojarad T, Roghani M, Khastehkhodaie Z. Chronic treatment of silymarin improves hyperalgesia and motor nerve conduction velocity in diabetic neuropathic rat. Phytother Res. 2010;24(8):1120-5.

29. You Z, Zheng Z, Blagg BSJ, Rick T. Dobrowsky.KU-596 Decreases Mitochondrial Superoxide and Improves Bioenergetics Following Downregulation of Manganese Superoxide Dismutase in Diabetic Sensory Neurons. Exp Neurol. 2019;313:88-97.

30. Wang R, Wang L, Zhang C, Zhang Y, Liu Y, Song L, Ma R, Dong J. L-carnitine ameliorates peripheral neuropathy in diabetic mice with a corresponding increase in insulin-like growth factor-1 level. Mol Med Rep. 2019:19:743-51.

31. Wu Y, Ye F, Lu Y, Yong H, Yin R, Chen B, Yong Y. Diosgenin glucoside protects against myocardial injury in diabetic mice by inhibiting RIP140 signaling. Am J Transl Res. 2018;10(11):3742-9.

32. Dong J, Zuo Z, Yan W, Liu W, Zheng Q, Liu X. Berberine ameliorates diabetic neuropathic pain in a rat model: involvement of oxidative stress, inflammation, and $\mu$-opioid receptors. Naunyn Schmiedeberg's Arch Pharmacol. 2019;392(9):1141-9.

33. Behse F, Buchthal F, Carlsen F. Nerve biopsy and conduction studies in diabetic neuropathy. J Neurol Neurosurg Psychiatry. 1977;40(11):1072-82.

34. Cameron NE, Eaton SE, Cotter MA, Tesfaye S. Vascular factors and metabolic interactions in the pathogenesis of diabetic neuropathy. Diabetologia. 2001; 44(11):1973-88.

35. Torres-Sanchez S, Borges GDS, Mico JA, Berrocoso E. Opioid and noradrenergic contributions of tapentadol to the inhibition of locus coeruleus neurons in the streptozotocin rat model of polyneuropathic pain. Neuropharmacology. 2018;135:202-10.

36. Ri-Ge-le A, Guo ZL, Wang Q, Zhang BJ, Kong DW, Yang WQ, Yu YB, Zhang L. Tanshinone IIA improves painful diabetic neuropathy by suppressing the expression and activity of voltage-gated Sodium Channel in rat dorsal root ganglia. Exp Clin Endocrinol Diabetes. 2018;126(10):632-9.

37. Etienne I, Magalhães LVB, Cardoso SA de Freitas RB, de Oliveira GP, Palotás A, Lima LM. Oxidative stress markers in cognitively intact patients with diabetic neuropathy. Brain Res Bull. 2019;150:196-200.

38. Areti A, Yerra VG, Naidu V, Kumar A. Oxidative stress and nerve damage: role in chemotherapy induced peripheral neuropathy. Redox Biol. 2014:2. 289-95.

39. Khosravi Z, Sedaghat R, Baluchnejadmojarad T, Roghani M. Diosgenin ameliorates testicular damage in streptozotocin-diabetic rats through attenuation of apoptosis, oxidative stress, and inflammation. Int Immunopharmacol. 2019:70:37-46.

40. Yang B, Xu B, Zhao H, Wang YB, Zhang J, Li CW, Wu Q, Cao YK, Li Y, Cao F. Dioscin protects against coronary heart disease by reducing oxidative stress and inflammation via Sirt1/Nrf2 and p38 MAPK pathways. Mol Med Rep. 2018;18(1):973-80

41. Inoguchi T, Li P, Umeda F, Yu HY, Kakimoto M, Imamura M, Aoki T, Etoh T, Hashimoto T, Naruse M, Sano H, Utsumi H. High glucose level and free fatty acid stimulate reactive oxygen species production through protein kinase C--dependent activation of $\mathrm{NAD}(\mathrm{P}) \mathrm{H}$ oxidase in cultured vascular cells. Diabetes. 2000;49(11):1939-45. 
42. Brownlee M. Biochemistry and molecular cell biology of diabetic complications. Nature. 2001;414(6865):813-20.

43. Singh R, Kishore L, Kaur N. Diabetic peripheral neuropathy: current perspective and future directions. Pharmacol Res. 2014;80:21-35.

44. Kasznicki J, Kosmalski M, Sliwinska A, Mrowicka M, Stanczyk M, Majsterek I, Drzewoski J. Evaluation of oxidative stress markers in pathogenesis of diabetic neuropathy. Mol Biol Rep. 2012;39(9):8669-78.

45. Afanas'ev I. Signaling of reactive oxygen and nitrogen species in diabetes mellitus. Oxidative Med Cell Longev. 2010;3(6):361-73.

46. Rahal A, Kumar A, Singh V, Yadav B, Tiwari R, Chakraborty S, Dhama K. Oxidative stress, prooxidants, and antioxidants: the interplay. Biomed Res Int. 2014;2014:761264.

47. Russell JW, Golovoy D, Vincent AM, Mahendru P, Olzmann JA, Mentzer A, Feldman EL. High glucose-induced oxidative stress and mitochondrial dysfunction in neurons. FASEB J. 2002:16(13):1738-48.

48. Peng S, Hou Y, Yao J, Fang J. Activation of Nrf2-driven antioxidant enzymes by cardamonin confers neuroprotection of $\mathrm{PC} 12$ cells against oxidative damage. Food FunctFood \& function. 2017;8(3):997-1007.

49. Negi G, Nakkina V, Kamble P, Sharma SS. Heme oxygenase-1, a novel target for the treatment of diabetic complications: focus on diabetic peripheral neuropathy. Pharmacol Res. 2015;102:158-67.

\section{Publisher's Note}

Springer Nature remains neutral with regard to jurisdictional claims in published maps and institutional affiliations.

Ready to submit your research? Choose BMC and benefit from:

- fast, convenient online submission

- thorough peer review by experienced researchers in your field

- rapid publication on acceptance

- support for research data, including large and complex data types

- gold Open Access which fosters wider collaboration and increased citations

- maximum visibility for your research: over $100 \mathrm{M}$ website views per year

At BMC, research is always in progress.

Learn more biomedcentral.com/submissions 